\title{
Red-to-Blue Photon Upconversion Enabled by One Dimensional CdTe Nanorods
}

Zachary A. VanOrman, Carl R. Conti III, Geoffrey F. Strouse, and Lea Nienhaus*

Department of Chemistry and Biochemistry, Florida State University, Tallahassee, FL 32306,

USA

*corresponding Author: $\underline{\text { lnienhaus @fsu.edu }}$ 


\section{ABSTRACT}

Photon upconversion via triplet-triplet annihilation creating light in the high energy regime of the visible spectrum could prove particularly useful in applications such as photocatalysis. Quantumconfined semiconductor nanocrystals have previously been shown to function as efficient triplet sensitizers in green-to-blue upconversion schemes, but an improvement in the apparent anti-Stokes shift of the upconversion scheme will be beneficial for future commercial applications. Additionally, both zero- and two-dimensional quantum-confined sensitizers have been investigated, but not one-dimensional nanomaterials. In this work, we fill a hole in the present field of photon upconversion by accomplishing both of these feats. Specifically, we introduce CdTe nanorods as a new class of triplet sensitizers for red-to-blue photon upconversion. When the triplet transmitter ligand (9-anthracenecarboxylic acid) and triplet annihilator (9,10-diphenylanthracene) are added to the nanorods, we observe efficient photon upconversion at a normalized upconversion efficiency of $\eta_{\mathrm{uc}}=4.3 \%$ and a low threshold power of $\mathrm{I}_{\mathrm{th}}=93 \mathrm{~mW} / \mathrm{cm}^{2}$. The introduction of onedimensional triplet sensitizers could yield future research that effectively harnesses the unique properties of these materials allowing for new approaches for efficient photon upconversion, especially at large apparent anti-Stokes shifts. 


\section{INTRODUCTION}

Photon upconversion (UC) via triplet-triplet annihilation (TTA) in organic semiconductors is an efficient way to generate higher energy photons at relatively low fluences. ${ }^{1}$ TTA-UC could prove to be useful in a multitude of applications, such as photocatalysis, ${ }^{2-4}$ optogenetics, ${ }^{5}$ photovoltaics ${ }^{6,7}$ or bioimaging. ${ }^{8}$ In lieu of metal-organic complexes with exchange energy losses on the order of hundreds of meVs, ${ }^{9}$ recently inorganic semiconductor materials including bulk perovskites ${ }^{10-13}$ and spherical semiconductor quantum dots (QDs) have emerged as efficient triplet sensitizers for the TTA-UC process. ${ }^{14-16}$

Additionally, quantum-confined semiconductor materials beyond QDs have been studied, namely two-dimensional (2D) nanoplatelets (NPLs), as these materials are less prone to parasitic effects stemming from size polydispersity. ${ }^{17}$ However, one dimensional (1D) materials, where two dimensions of the nanomaterial are quantum-confined, have not yet been investigated as potential triplet sensitizers. Here, we introduce cadmium telluride (CdTe) nanorods (NRs) as triplet sensitizers for anthracene-based upconverters.

1D nanomaterials, such as NRs, and nanowires, have been previously studied for a multitude of applications, including lasing, ${ }^{18}$ photonics, ${ }^{19}$ bioelectronics, ${ }^{20}$ and photovoltaics. ${ }^{21}$ The welldefined facets and unique anisotropic shape of 1D nanomaterials also allow for self-assembly into macrostructures, ${ }^{22}$ which could be useful for photon UC, especially in the solid state. ${ }^{23,24}$ In-depth studies of the unique properties of 1D materials endowed by their aspect ratio and anisotropic nature are required pertaining to their application as triplet sensitizers in photon UC via TTA. These NRs not only fill in a gap of the material dimensionality of existing triplet sensitizers, but also in the energetic regime of UC under study. While green-to-blue ${ }^{14,25,26}$ and blue-to-UV UC schemes ${ }^{27-29}$ are useful for photocatalytic applications, it could also be beneficial to extend the 
apparent Anti-Stokes shift of the TTA-UC system, yielding a system capable of red-to-blue UC or beyond.

Recent works have realized red-to-blue UC using silicon $\mathrm{QDs}^{30}$ and cadmium selenide (CdSe) QDs as triplet sensitizers. ${ }^{31}$ However, silicon QDs exhibit relatively low photoluminescent quantum yields (PLQY), and red-emitting CdSe QDs are on the edge of the quantum confinement regime, which has been shown to have negative impacts on the efficiency of the UC process. ${ }^{26,32,33}$ We take advantage of CdTe, which allows for redder emission at smaller nanocrystal sizes as compared to CdSe. ${ }^{34}$ The as-synthesized NRs can be paired with a transmitter ligand and a triplet annihilator to realize efficient red-to-blue UC. We use 9-anthracene carboxylic acid (ACA) as the triplet transmitter ligand and 9,10-diphenylanthracene (DPA) as the blue-emitting triplet annihilator. ACA has previously been used as a transmitter ligand for CdSe QD sensitized UC schemes, ${ }^{14,26}$ where the TET process has been shown to occur through space. ${ }^{35}$

Here, we observe efficient red-to-blue TTA-UC with UC efficiencies in excess of $4 \%$ with an apparent anti-Stokes shift of $0.82 \mathrm{eV}$. Additionally, we observe a power threshold for efficient UC of $\mathrm{I}_{\mathrm{th}}=93 \mathrm{~mW} / \mathrm{cm}^{2}$, which is considerably lower than the majority of systems utilizing quantumconfined triplet sensitizers. ${ }^{17,25,31,36}$ Most importantly, we open the door to a new frontier of triplet sensitizer materials in the form of 1D semiconductor nanocrystals.

\section{RESULTS/DISCUSSION}

CdTe NRs were synthesized by injecting $36 \mathrm{mg}$ of Te in $3 \mathrm{~mL}$ of trioctylphosphine (TOP) into a Cd-oleate solution at $200{ }^{\circ} \mathrm{C}$ (see Experimental Methods). Upon purification with acetone and methanol, the NRs were resuspended in hexanes. As shown in the scanning transmission electron microscopy (STEM) image in Figure 1a, the NRs are slightly curved in appearance, with a radius 
of $\sim 3 \mathrm{~nm}$. Additional high-resolution STEM images are shown in Figure S1. Figure 1b shows the normalized absorbance and PL spectrum of the NRs in hexanes, where the emission peak maximum is at $649 \mathrm{~nm}$, slightly Stokes-shifted from the maximum absorbance of $\sim 612 \mathrm{~nm}$.
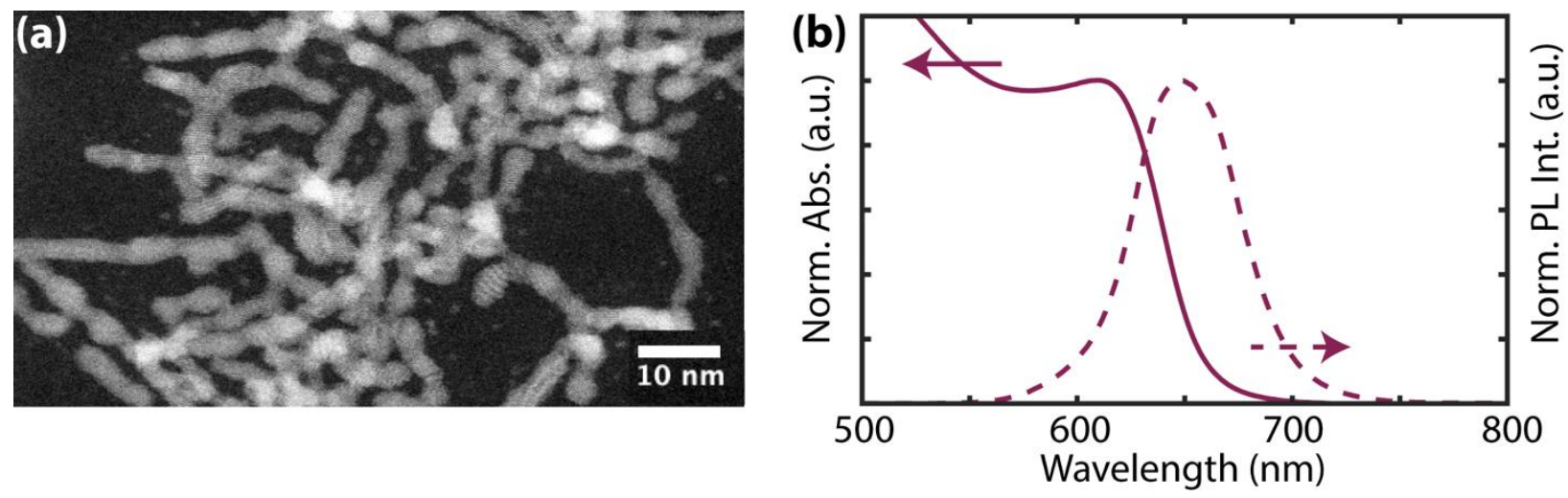

Figure 1:_CdTe nanorod (NR) characterization. a) Scanning transmission electron microscopy (STEM) image of the CdTe NRs. b) Normalized absorbance and PL emission of the CdTe NRs. The PL emission of the NRs was monitored under $520 \mathrm{~nm}$ excitation, where a $550 \mathrm{~nm}$ long-pass filter was used to remove excess laser scatter.

Additionally, we note that the appearance of the emission peak changes upon stirring the NRs vigorously overnight. While the absorption spectrum does not change in the stirred NRs, the emission peak maximum blue shifts to $\sim 633 \mathrm{~nm}$ (Figure S2). We attribute this to resonance energy transfer between aggregated NRs, where the blue edge of the emission spectrum appears to be quenched in the unstirred sample..$^{37,38}$

In order to obtain efficient red-to-blue TTA-UC, in addition to the triplet sensitizer, both a triplet mediator or transmitter ligand, ${ }^{14,39}$ and a triplet annihilator are required. The overall energetic scheme of the TTA-UC system is shown in Figure 2a. Upon optical excitation via absorption of an incident photon, an electron is excited from the valence band to the conduction band in the CdTe NR. The spin-triplet state of the ACA transmitter ligand can then be populated via a triplet energy transfer (TET) following a Dexter-type exchange mechanism, ${ }^{40}$ which is energetically favorable in this case, as the NR excitonic transition is higher in energy than the ACA triplet level 
$\mathrm{T}_{1}=1.83 \mathrm{eV} .{ }^{14} \mathrm{~A}$ second triplet energy transfer $\left(\mathrm{TET}_{2}\right)$ can then occur to DPA, which has a $\mathrm{T}_{1}$ energy level of $1.77 \mathrm{eV}$. Once two DPA triplet states are occupied, and the two triplet-excited molecules collide, TTA-UC can occur. Upon relaxation of the resultant singlet excited state, a blue photon is emitted correlated to the $S_{1}$ energy level of DPA at $3.06 \mathrm{eV}$. The normalized absorbance and PL emission spectra for the relevant transmitter ligand, ACA, and triplet annihilator, DPA, are shown in Figure 2b. The UC emission of the ternary NR-ACA-DPA UC system will manifest as emission from the DPA after the TTA-UC process has occurred. Therefore, taken from the absorbance maximum of the sensitizer and the emission maximum of the annihilator, the apparent anti-Stokes shift of the system presented here is $0.82 \mathrm{eV},{ }^{41}$ which is comparable to recently reported red-to-blue QD sensitized UC schemes. ${ }^{31,42}$

The ligand exchange process occurs through the addition of ACA in chlorobenzene and allowing the solutions to stir overnight. This process not only helps to promote a dynamic ligand exchange environment between the oleate-capped NRs and the ACA but also helps to suppress the aggregation of the NRs discussed previously. 

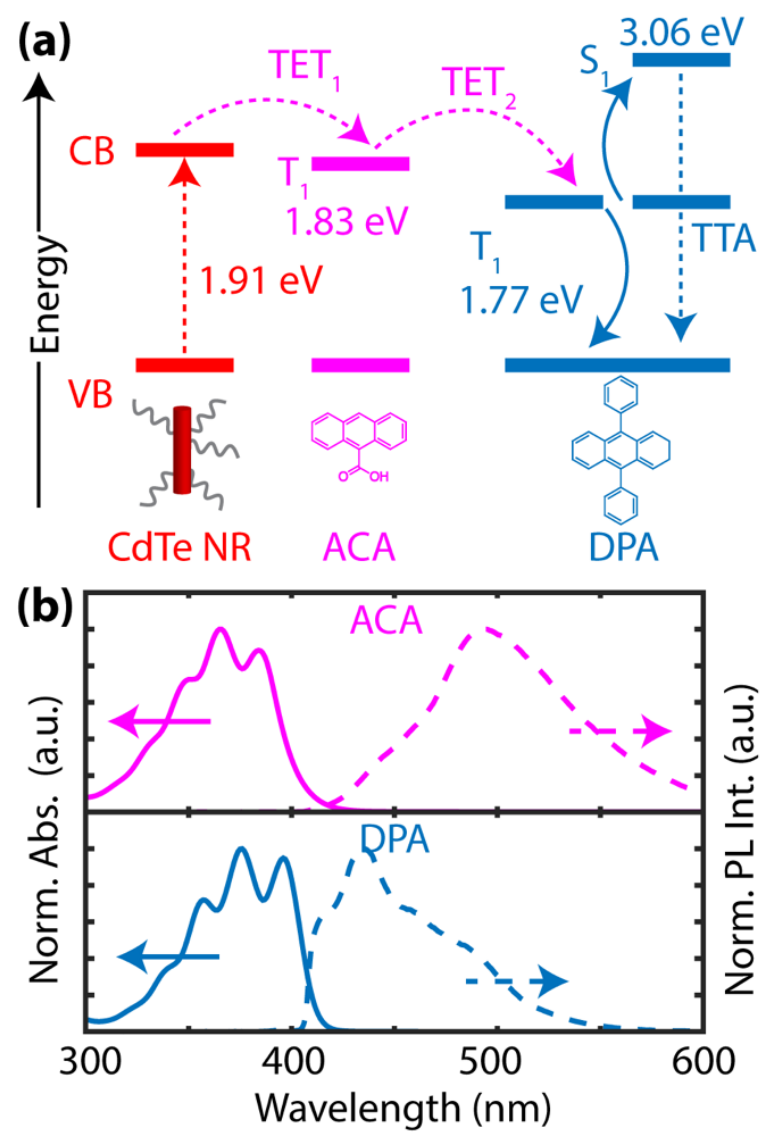

Figure 2: The proposed CdTe NR sensitized UC system. a) Schematic of the energy levels involved in the UC process, including the CdTe NR sensitizer bandgap (red), ACA triplet energy level (magenta), and DPA triplet and singlet state energies (blue). b) The normalized absorbance (solid) and PL emission (dashed) of ACA (top, magenta) and DPA (bottom, blue).

Here, we utilize a combination of ultrafast transient absorption (TA) and time-resolved photoluminescence (PL) spectroscopy in the form of time-correlated single-photon counting (TCSPC) to unravel the energy transfer process between the NRs and surface-bound ACA. The TA spectrum of NRs with their native ligand shell is shown in Figure 3a, where four distinct features are shown, corresponding to excitonic transitions at different energies. ${ }^{43}$ Most notable is the ground state exciton bleach (XB) at $635 \mathrm{~nm}$ due to state filling in the NRs. ${ }^{44}$ Additionally, two photoinduced absorption (PIA) features can be discerned at 550 and $487 \mathrm{~nm}$, where PIA features arise due to shifts in excited state energy levels. ${ }^{44}$ An additional bleach can be found at $435 \mathrm{~nm}$. To observe the change in the NR TA features after ligand exchange with ACA, the ultrafast TA 
measurements were repeated under the same conditions for the NR-ACA system (Figure 3b). Additionally, a sample with a concentration of ACA 20 times higher is shown in Figure S3a. Other studies have shown that upon triplet sensitization of ACA, an excited state absorption (ESA) feature centered at $\sim 435 \mathrm{~nm}$ can be observed upon excitation of the QD, corresponding to the ACA $T_{1}-T_{n}$ transition. ${ }^{42,45,46}$ However, this is not observed in Figure $3 b$, as the high energy NR bleach feature is also centered at $435 \mathrm{~nm}$, which obscures the ACA ESA peak. In order to observe the ACA ESA, double difference spectra were obtained by subtracting the CdTe NR spectrum from the NR-ACA spectra after normalizing the spectra to the $550 \mathrm{~nm}$ PIA feature at the 1 ps delay time. The double difference spectra for either ACA concentration are shown in Figure S4, where the growth of the ACA ESA can be observed, which occurs at a similar timescale to the bleach feature observed at $\sim 560 \mathrm{~nm}$, which is indicative of the sensitization of the triplet state in ACA from the CdTe NRs. Additionally, the kinetics of the PIA feature at $550 \mathrm{~nm}$ are shown in Figure 3c, where the PIA lifetime is quenched upon the addition of ACA. Furthermore, the kinetics of the PIA feature at $487 \mathrm{~nm}$, as well as the two bleach features, the XB at $635 \mathrm{~nm}$, and the high energy bleach feature at $435 \mathrm{~nm}$ are shown in Figure S3, where the same trend is observed, i.e., the lifetime is shortened upon a higher concentration of the ACA transmitter ligand. We emphasize that the XB kinetics are convoluted by the pump signal $(625 \mathrm{~nm})$ and that the bleach at $435 \mathrm{~nm}$ contains contributions from the ACA ESA.

The effect of the ACA coupling on the NR in both steady-state and time-resolved emission was also monitored. Figure S5 shows the effect of the time-resolved emission, monitored via TCSPC, and the steady-state emission of the NR upon addition of ACA. In both cases, the emission is significantly quenched. To further describe the degree of quenching of the NR emission, we plot 
in Figure 3d, both the quenching of the PL lifetime $\left(\tau_{0} / \tau\right)$ and of the steady-state emission $\left(\mathrm{I}_{0} / \mathrm{I}\right)$, in accordance with the Stern-Volmer equation, given in Equation 1.

$$
\frac{\tau_{0}}{\tau}=1+K_{S V}[Q]
$$

$\mathrm{K}_{\mathrm{SV}}$ corresponds to the Stern-Volmer constant, which is the product of the bimolecular quenching constant $\mathrm{k}_{\mathrm{q}}$ and the native lifetime $\tau_{0}$, and [Q] is the concentration of the quenching species, which, here, is the concentration of ACA. We observe a linear behavior from the quenching of the lifetime and a quadratic behavior for the quenching of the steady-state emission, which indicates the presence of both static and dynamic quenching, in line with similar behavior observed in OD CdSe QDs and 2D CdSe nanoplatelets. ${ }^{17}$ From the Stern-Volmer plot (with concentrations $<0.10 \mathrm{mM}$ ACA), we extract a bimolecular quenching constant of $8.82 \times 10^{12} \mathrm{M}^{-1} \mathrm{~S}^{-1}$ (See Supporting Information for more information). Above a concentration of $\sim 0.15 \mathrm{mM}$ ACA, a saturation of the quenching is observed, which is highlighted in Figure $3 \mathrm{~d}$ by a gray box. 

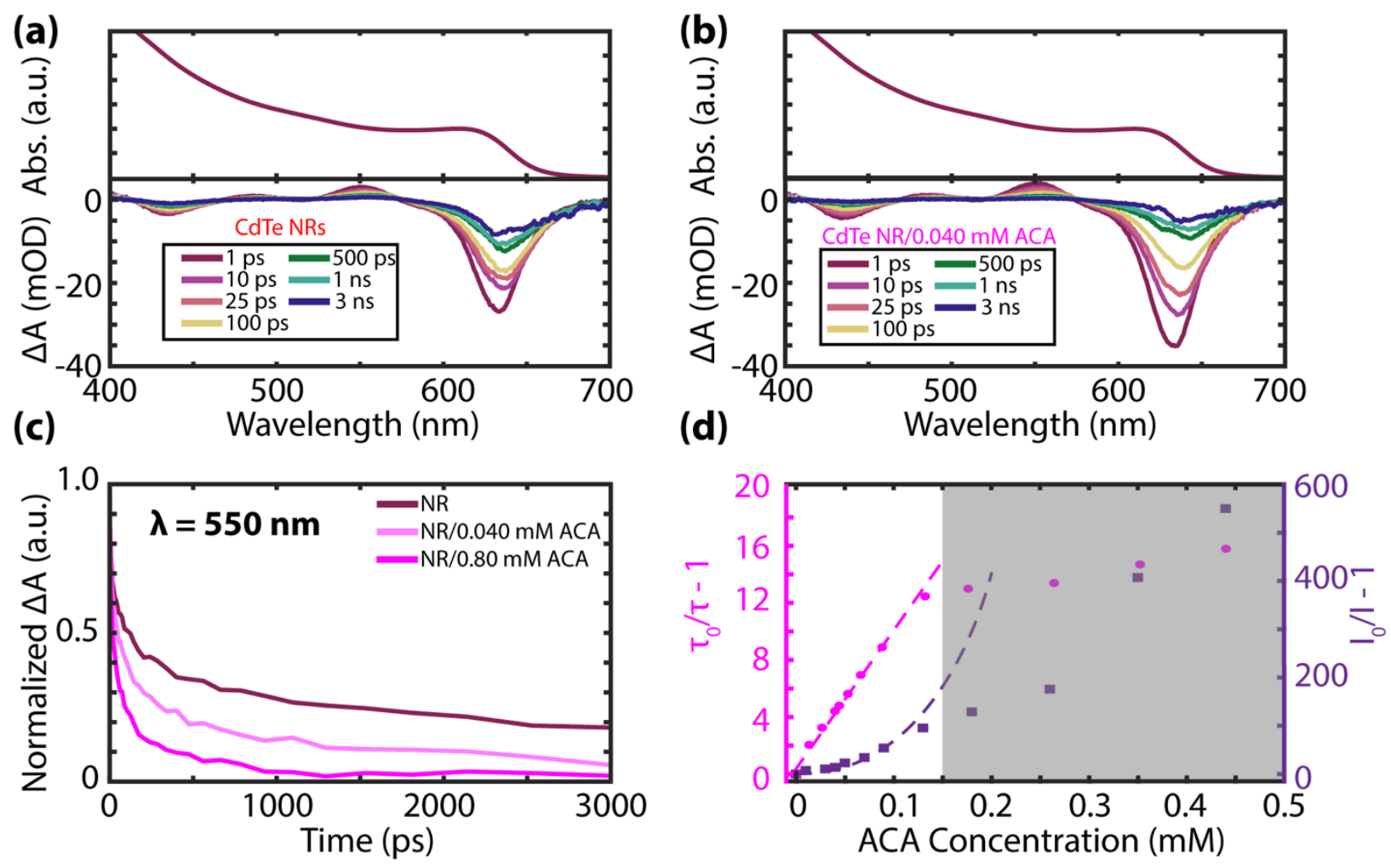

Figure 3: Ultrafast transient absorption (TA) spectra of a) the native CdTe NRs and b) NRs with $0.040 \mathrm{mM}$ ACA at various delay times after excitation at the band edge $(625 \mathrm{~nm})$. c) TA kinetics of the PIA peak at $550 \mathrm{~nm}$ for the NRonly (red), NR with $0.040 \mathrm{mM}$ ACA (light magenta) and $0.80 \mathrm{mM}$ ACA (magenta). d) Stern-Volmer plots of the NR lifetime (magenta, circles) and the steady-state emission intensity (purple, squares). The gray region denotes an apparent saturation point of the quenching.

The addition of DPA to NRs coupled with ACA results in a ternary system capable of TTA-UC, which can easily be observed by eye, as shown by the photographs in Figure 4a. When there is no ACA, little UC can be observed, and a cuvette containing NRs and DPA emits mostly red light upon $520 \mathrm{~nm}$ excitation due emission from the NRs. However, if the NRs are complexed with ACA before the DPA is added, blue emission from the $S_{1}$ state of DPA can be observed.

The emission spectrum of the NR-ACA-DPA system is shown in Figure 4b, where the $520 \mathrm{~nm}$ excitation results in emission from the NR, but also from the DPA annihilator. The region from 500-540 nm was removed from the PL spectrum due to excess laser scatter. The UC emission spectrum matches that which is observed upon direct excitation of DPA at $405 \mathrm{~nm}$, shown in Figure 
2b. The intensity of the upconverted emission follows a power dependence characteristic of TTAexhibiting systems, which is shown in a log-log plot of the UC PL intensity against the incident power in Figure 4c. At low powers, the upconverted PL increases quadratically with the incident power, in the regime where triplet decay pathways other than TTA are dominant, which yields a slope, $\alpha$ of $\sim 2$ in a log-log plot. ${ }^{47}$ At high powers, where TTA is the dominant triplet decay pathway, the upconverted PL increases linearly with the incident power, yielding $\alpha \sim 1$.

The point at which these regimes meet gives the threshold intensity value $\mathrm{I}_{\text {th }}$, which is a figure of merit for the power density at which the TTA-UC process becomes efficient. For the system with the most efficient TTA-UC, at an ACA concentration of $0.04 \mathrm{mM}$ ACA, an $\mathrm{I}_{\text {th }}$ value of $93 \mathrm{~mW} / \mathrm{cm}^{2}$ was determined, which is considerably lower compared to many other quantum confined semiconductor sensitized systems, ${ }^{12,15,17,28,29,36,48}$ especially those that exhibit red-to-blue UC. ${ }^{31,42}$ Only the upconverted emission follows this unique power dependence and the excitonic NR emission exhibits the expected linear regime at all powers, shown in Figure S6.

Additionally, the UC efficiency, notated as $\eta_{\mathrm{uc}}$, was measured relative to a rubrene standard for different ACA concentrations, which is shown in Figure 4c. Here, we choose to normalize the UC quantum yield by two, as TTA is a two-photon to one process, meaning the upper limit of the UC quantum yield (QY) is $50 \% .^{41}$ The $\eta_{\text {uc }}$ was calculated according to Castellano and coworkers, ${ }^{1}$ where the absorbance and UC emission of the NR-ACA-DPA system was compared to that of rubrene, which is shown in Figure S7, in accordance to Equation 2.

$$
\eta_{u c}=2 \frac{\Phi_{r u b}}{\Phi_{D P A}}\left(\frac{A_{r u b}}{A_{U C}}\right)\left(\frac{I_{U C}}{I_{r u b}}\right)\left(\frac{n_{U C}}{n_{r u b}}\right)^{2}
$$

$\Phi_{\text {rub }}$ is the PL QY of rubrene, which is divided by the QY of DPA, as the annihilator QY is not at unity, A reflects the absorption of either the rubrene standard or the UC system at the excitation wavelength of $520 \mathrm{~nm}, \mathrm{I}$ is the integrated emission spectrum and $\mathrm{n}$ corresponds to the refractive 
index of either system (for more detail, see Supporting Information). Only faint UC is observed without the presence of ACA, and the $\eta_{\mathrm{uc}}$ increases to a maximum of $\eta_{\mathrm{uc}}=4.3 \%$ at an optimal ACA concentration of $0.04 \mathrm{mM}$ and then decreases sharply (compare Table S1).

As expected, we observe that the ACA ligand concentrations which result in higher $\eta_{\text {uc }}$ values also yield lower Ith values, as shown in Figure S6. Both the NR-DPA system and a NR-ACA-DPA UC system with an ACA concentration much higher than the optimal concentration have $\mathrm{I}_{\text {th }}$ values near $1 \mathrm{~W} / \mathrm{cm}^{2}$, nearly ten times higher than that of the optimal concentration of $0.04 \mathrm{mM}$. At concentrations closer to the optimal concentration of $0.04 \mathrm{mM}$ (Figure S6d), the $\mathrm{I}_{\text {th }}$ begins to decrease toward the lowest $\mathrm{I}_{\mathrm{th}}$ of $93 \mathrm{~mW} / \mathrm{cm}^{2}$. While it is simpler to imagine the lower $\eta_{\text {uc }}$ stemming from less bound ACA, i.e., concentrations below $0.04 \mathrm{mM}$, the decline of $\eta_{\mathrm{uc}}$ above the optimal ACA concentration has also been previously observed in CdSe QD sensitized systems. ${ }^{42,48,49}$

The cause of this decline in the $\eta_{u c}$ above the optimal concentration has not yet been completely unraveled in the previously reported QD-sensitized systems and cannot yet be attributed to a certain process in the NR-sensitized UC presented here and will be the focus of further investigations. For example, the role of the internal grain boundaries where nanoparticles were fused to obtain the elongated NRs in the upconversion mechanism is unclear and is of future interest. It is possible that these act as undesired recombination centers, and thus result in a decrease of the possible UC efficiency. In addition, in the UC system presented here, the likelihood of aggregation of the 1D NRs introduces another potential loss pathway analogous to nanoplatelet stacking, which has been observed previously in nanoplatelet-sensitized TTA-UC. ${ }^{17}$ 
(a)
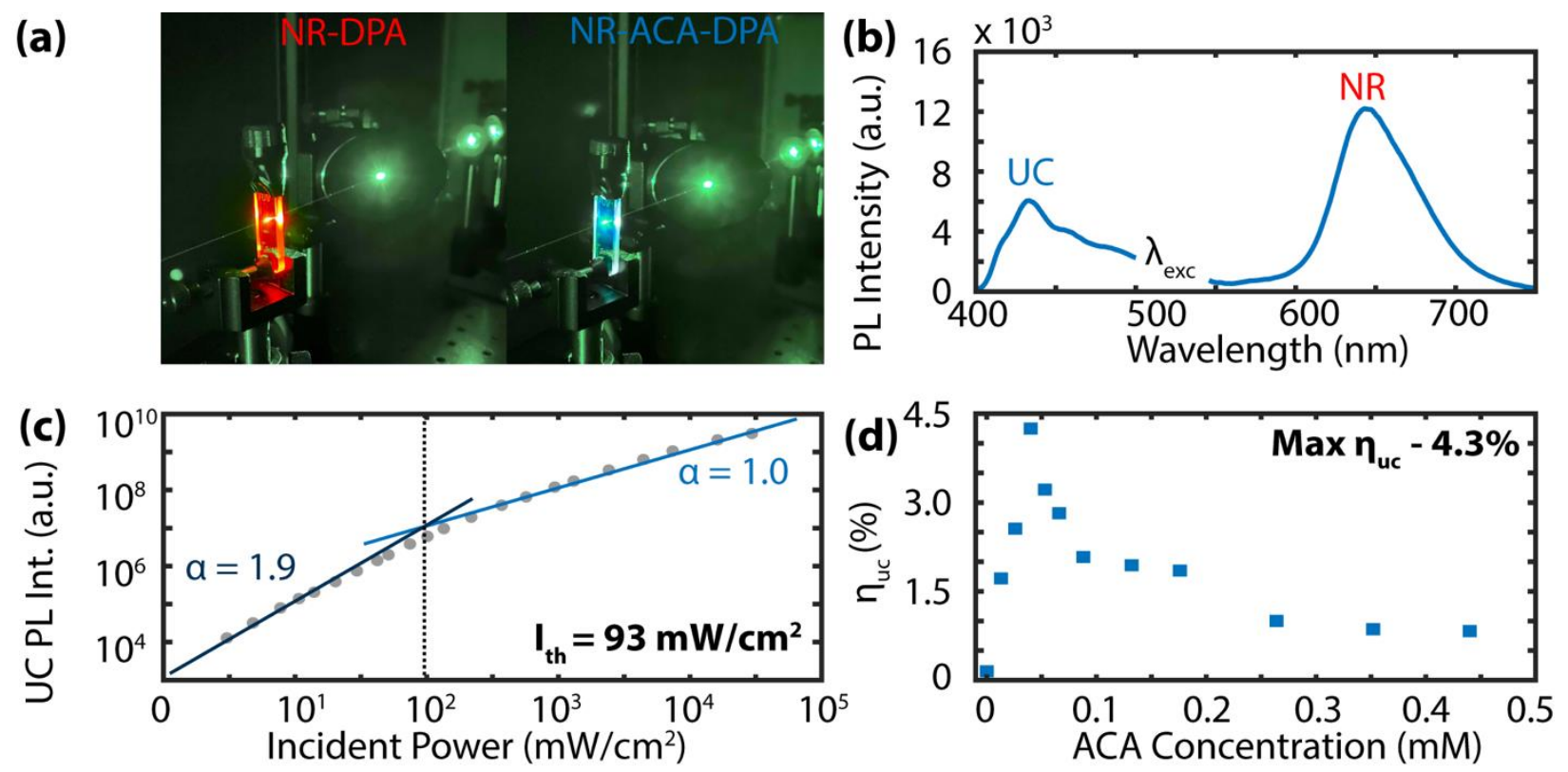

Figure 4: Characterization of the CdTe NR sensitized UC process. a) Photograph of the NR-DPA system (left) without ACA and the NR-ACA-DPA UC system (right), where the blue emission can be observed as a result of TTA-UC. b) Steady state emission of the NR-ACA-DPA system under $520 \mathrm{~nm}$ excitation. The region from $500-540 \mathrm{~nm}$ was removed due to excess laser scatter. c) Log-log plot of the UC PL intensity against the incident power applied to the NR-ACA-DPA UC system with $0.04 \mathrm{mM}$ ACA yielding an $\mathrm{I}_{\mathrm{th}}=93 \mathrm{~mW} / \mathrm{cm}^{2}$. d) The UC efficiency, $\eta_{\mathrm{uc}}$, plotted against the ACA concentration. The maximum $\eta_{\mathrm{uc}}=4.3 \%$ is observed at a concentration of $0.04 \mathrm{mM}$ ACA.

In conclusion, we have shown that CdTe NRs are viable triplet sensitizers for use in red-to-blue UC schemes, which is specifically reflected by the $\eta_{\mathrm{uc}}$ exceeding $4 \%$ and the $\mathrm{I}_{\mathrm{th}}$ value below 100 $\mathrm{mW} / \mathrm{cm}^{2}$ at an optimal ACA concentration of $0.04 \mathrm{mM}$. Additionally, this work fills a current gap in literature by introducing 1D triplet sensitizers for TTA-UC, which represent a new avenue for triplet sensitizer design in TTA-UC. The rich knowledge of 1D semiconductor nanomaterials gleaned from other applications applied to the field of photon upconversion could allow for new frontiers to be uncovered. Beyond the scope of the work presented here, further investigation of the loss mechanisms occurring at higher ACA concentrations will allow for a better understanding of the energy transfer processes, which can translate into even higher UC efficiencies, pushing TTA-UC toward their eventual industrial relevance. 


\section{EXPERIMENTAL METHODS:}

\section{Materials}

Tellerium (30 mesh), trioctylphosphine (TOP), cadmium oxide, oleic acid, octadecene, methanol, hexanes, chlorobenzene, toluene, and rubrene were purchased from Millipore Sigma. 9Anthracenecarboxylic acid (ACA) and 9,10-diphenylanthracene (DPA) were purchased from TCI Chemicals. Acetone was purchased from Fisher. All chemicals were used as received without further modification.

\section{Te-TOP Preparation}

Te-TOP solution was made by adding $36 \mathrm{mg}$ of Te powder to $3 \mathrm{~mL}$ of TOP. The mixture was lightly heated at $35^{\circ} \mathrm{C}$ and stirred for $1 \mathrm{hr}$ in a glovebox under air-free conditions.

\section{CdTe Nanorod Synthesis}

CdTe nanorods (NRs) were synthesized by first adding $120 \mathrm{mg}$ of $\mathrm{CdO}, 1.33 \mathrm{~mL}$ of oleic acid, and $15 \mathrm{~mL}$ of octadecene into a $25 \mathrm{~mL}$ 3-neck flask. The mixture was degassed under vacuum at 120 ${ }^{\circ} \mathrm{C}$ for 1 hour. The mixture was then put under $\mathrm{N}_{2}$ gas and brought to $200{ }^{\circ} \mathrm{C}$. After the solution became clear, $3 \mathrm{~mL}$ of Te-TOP was injected quickly. The reaction mixture was maintained at 200 ${ }^{\circ} \mathrm{C}$ for 5 minutes and then was allowed to cool in air. The NRs were quickly transferred to a $\mathrm{N}_{2}$ atmosphere glovebox for purification.

\section{CdTe Nanorod Purification}

$15 \mathrm{~mL}$ of a mixture of acetone and methanol were added to the NR solution before centrifugation at $6000 \mathrm{rpm}$ for 15 minutes. The pellet was then redispersed in $1 \mathrm{~mL}$ hexanes.

\section{Ligand Exchange}

From the $1 \mathrm{~mL}$ hexanes concentrated NR solution, $50 \mu \mathrm{L}$ were diluted to $12 \mathrm{~mL}$ to make a NR stock solution. Various amounts of ACA and chlorobenzene were added to $200 \mu \mathrm{L}$ of the NR stock 
solution, the ACA in chlorobenzene was $3.08 \mathrm{mM}$. In all cases, the NR concentration and the amount of chlorobenzene added was kept constant.

\section{UC Sample Preparation}

For UC measurements, $150 \mu \mathrm{L}$ of a $10 \mathrm{mg} / \mathrm{mL}$ DPA in chlorobenzene was added to $200 \mu \mathrm{L}$ of NRACA solution. The samples were prepared in the glovebox and the $1 \mathrm{~mm}$ pathlength cuvettes were sealed with electrical tape.

\section{Methods}

\section{Transmission Electron Microscopy}

Scanning transmission electron microscopy (STEM) images were measured by using a JEOL-JEM ARM200cF microscope.

Steady-State Optical Characterization

UV-Visible absorption spectra were measured by a Thermo Scientific Evolution 220 Spectrophotometer. Steady-state emission spectra were monitored via an Ocean Optics spectrometer (HR2000+ES) under either $405 \mathrm{~nm}$ continuous wave (CW) (LDH-D-C-405, PicoQuant) or $520 \mathrm{~nm} \mathrm{CW}$ (LDH-D-C-520, PicoQuant) excitation. In the case of $405 \mathrm{~nm}$ excitation, excess laser scatter was removed by a $425 \mathrm{~nm}$ long pass filter (ThorLabs). For NR-only steady state emission, excess laser scatter was removed via a $550 \mathrm{~nm}$ long pass filter (ThorLabs). For UC steady-state emission spectra, no filter was used to monitor both the NR and UC emission.

\section{Ultrafast Transient Absorption}

Transient absorption was performed using a HELIOS Fire transient absorption spectrometer (Ultrafast Systems). Femtosecond pulses were generated using a Coherent Astrella-V-F-1K amplifier system. A Vitara-S Coherent Ti:sapphire laser was amplified using a $1 \mathrm{kHz}$ Coherent Revolution-50 pump laser and generated a $5 \mathrm{~mJ}$ pulse with a $100 \mathrm{fs}$ full width half max at $800 \mathrm{~nm}$. 
The pulse was split into a pump and a probe beam, where the probe was directed through a delay stage, while the pump passed through an optical parametric amplifier (OPerA Solo, Coherent). The white light continuum used for the probe was produced by a $\mathrm{CaF}_{2}$ crystal for all measurements. The pump and probe beam were then overlapped on the solution sample, which was previously sealed in a $1 \mathrm{~mm}$ pathlength UV quartz cuvette (Starna Cells) inside of an inert atmosphere glovebox. Difference spectra and single wavelength kinetics were collected averaging 3 times and holding for 3 seconds, with an exponential point acquisition beginning with 0.01 ps steps and totaling to 100 points. Data was processed using the Surface Xplorer software package from Ultrafast Systems.

Time-correlated Single Photon Counting (TCSPC)

PL lifetimes were collected by TCPSC as detailed previously. ${ }^{11,17,50}$ Lifetimes were measured under $520 \mathrm{~nm}$ excitation at a repetition frequency of $1 \mathrm{MHz}$. Photon arrival times were histogrammed by a MultiHarp 150 (PicoQuant) at a single photon counting avalanche diode (Micron Photon Devices). All lifetimes were measured at a power of $15 \mathrm{~mJ}$. Ith measurements were conducted under similar conditions, however the $520 \mathrm{~nm}$ laser was operated at CW and a $500 \mathrm{~nm}$ short pass filter (ThorLabs) was applied to remove laser scatter and the NR emission.

\section{ASSOCIATED CONTENT}

\section{Supporting Information.}

Stern-Volmer quenching calculations information, upconversion efficiency calculations, and supplemental figures: tabulated lifetimes and $\eta_{\mathrm{uc}}$ for CdTe NRs with various concentrations of ACA (Table S1); additional high resolution scanning transmission electron microscopy (STEM) images (Figure S1); UV-visible absorption and PL emission spectra of stirred and unstirred CdTe 
NRs (Figure S2); ultrafast TA spectrum of a NR-0.80 mM ACA solution at various delay times, along with other kinetic decay traces from various features in the TA spectra (Figure S3); Doubledifference TA spectra at two different ACA concentrations (Figure S4); NR time-resolved and steady-state PL emission quenching upon addition of ACA (Figure S5); Ith plots of the NR only emission, along with UC Ith plots with various ACA concentrations (Figure S6); UV-Vis absorption and PL emission of the rubrene standard (Figure S7).

\section{AUTHOR INFORMATION}

\section{Corresponding Author}

*E-mail: lnienhaus@fsu.edu

\section{Notes}

The authors declare no competing financial interests.

\section{ACKNOWLEDGEMENT}

Z.A.V. and L.N. gratefully acknowledge Florida State University startup funds, as well as a planning grant from the council on research and creativity (CRC) at FSU. C.R.C. and G.F.S. gratefully acknowledge the National Science Foundation under Grant No. DMR-1905757. We thank Yan Xin for STEM measurements. A portion of this work was performed at the National High Magnetic Field Laboratory, which is supported by the National Science Foundation Cooperative Agreement No. DMR-1644779 and the State of Florida. Transient absorption measurements were performed on a spectrometer supported by the National Science Foundation under Grant No. CHE-1919633. 


\section{REFERENCES}

(1) Singh-Rachford, T. N.; Castellano, F. N. Photon Upconversion Based on Sensitized TripletTriplet Annihilation. Coord. Chem. Rev. 2010, 254 (21-22), 2560-2573. https://doi.org/10.1016/j.ccr.2010.01.003.

(2) Khnayzer, R. S.; Blumhoff, J.; Harrington, J. A.; Haefele, A.; Deng, F.; Castellano, F. N. Upconversion-Powered Photoelectrochemistry. Chem. Commun. 2011, 48 (2), 209-211. https://doi.org/10.1039/C1CC16015J.

(3) Ravetz, B. D.; Pun, A. B.; Churchill, E. M.; Congreve, D. N.; Rovis, T.; Campos, L. M. Photoredox Catalysis Using Infrared Light via Triplet Fusion Upconversion. Nature 2019, 565 (7739), 343. https://doi.org/10.1038/s41586-018-0835-2.

(4) Pfund, B.; Steffen, D. M.; Schreier, M. R.; Bertrams, M.-S.; Ye, C.; Börjesson, K.; Wenger, O. S.; Kerzig, C. UV Light Generation and Challenging Photoreactions Enabled by Upconversion in Water. J. Am. Chem. Soc. 2020. https://doi.org/10.1021/jacs.0c02835.

(5) Sasaki, Y.; Oshikawa, M.; Bharmoria, P.; Kouno, H.; Hayashi-Takagi, A.; Sato, M.; Ajioka, I.; Yanai, N.; Kimizuka, N. Near-Infrared Optogenetic Genome Engineering Based on Photon-Upconversion Hydrogels. Angewandte Chemie International Edition 2019, 58 (49), 17827-17833. https://doi.org/10.1002/anie.201911025.

(6) Schulze, T. F.; Schmidt, T. W. Photochemical Upconversion: Present Status and Prospects for Its Application to Solar Energy Conversion. Energy Environ. Sci. 2014, 8 (1), 103-125. https://doi.org/10.1039/C4EE02481H.

(7) Ferreira, R. A. S.; Correia, S. F. H.; Monguzzi, A.; Liu, X.; Meinardi, F. Spectral Converters for Photovoltaics - What's Ahead. Materials Today 2020, 33, 105-121. https://doi.org/10.1016/j.mattod.2019.10.002.

(8) Askes, S. H. C.; Pomp, W.; Hopkins, S. L.; Kros, A.; Wu, S.; Schmidt, T.; Bonnet, S. Imaging Upconverting Polymersomes in Cancer Cells: Biocompatible Antioxidants Brighten Triplet-Triplet Annihilation Upconversion. Small 2016, 12 (40), 5579-5590. https://doi.org/10.1002/smll.201601708.

(9) Amemori, S.; Sasaki, Y.; Yanai, N.; Kimizuka, N. Near-Infrared-to-Visible Photon Upconversion Sensitized by a Metal Complex with Spin-Forbidden yet Strong S0-T1 Absorption. J. Am. Chem. Soc. 2016, $138 \quad$ (28), 8702-8705. https://doi.org/10.1021/jacs.6b04692.

(10) Nienhaus, L.; Correa-Baena, J.-P.; Wieghold, S.; Einzinger, M.; Lin, T.-A.; Shulenberger, K. E.; Klein, N. D.; Wu, M.; Bulović, V.; Buonassisi, T.; Baldo, M. A.; Bawendi, M. G. TripletSensitization by Lead Halide Perovskite Thin Films for near-Infrared-to-Visible Upconversion. ACS Energy Lett. 2019, 4 (4), 888-895. https://doi.org/10.1021/acsenergylett.9b00283.

(11) Wieghold, S.; Bieber, A. S.; VanOrman, Z. A.; Daley, L.; Leger, M.; Correa-Baena, J.-P.; Nienhaus, L. Triplet Sensitization by Lead Halide Perovskite Thin Films for Efficient SolidState Photon Upconversion at Subsolar Fluxes. Matter 2019, 1 (3), 705-719. https://doi.org/10.1016/j.matt.2019.05.026. 
(12) VanOrman, Z. A.; Bieber, A. S.; Wieghold, S.; Nienhaus, L. A Perspective on Triplet Fusion Upconversion: Triplet Sensitizers beyond Quantum Dots. MRS Communications 2019, 9 (3), 924-935. https://doi.org/10.1557/mrc.2019.115.

(13) Wieghold, S.; Nienhaus, L. Engineering 3D Perovskites for Photon Interconversion $\begin{array}{llllll}\text { Applications. } & \text { PLOS } & \text { ONE } & \text { 2020, } & 15 & \text { (3), }\end{array}$ https://doi.org/10.1371/journal.pone.0230299.

(14) Huang, Z.; Li, X.; Mahboub, M.; Hanson, K. M.; Nichols, V. M.; Le, H.; Tang, M. L.; Bardeen, C. J. Hybrid Molecule-Nanocrystal Photon Upconversion across the Visible and near-Infrared. Nano Lett. 2015, $15 \quad$ (8), 5552-5557. https://doi.org/10.1021/acs.nanolett.5b02130.

(15) Okumura, K.; Mase, K.; Yanai, N.; Kimizuka, N. Employing Core-Shell Quantum Dots as Triplet Sensitizers for Photon Upconversion. Chem. Eur. J. 2016, 22 (23), 7721-7726. https://doi.org/10.1002/chem.201600998.

(16) Wu, M.; Congreve, D. N.; Wilson, M. W. B.; Jean, J.; Geva, N.; Welborn, M.; Van Voorhis, T.; Bulović, V.; Bawendi, M. G.; Baldo, M. A. Solid-State Infrared-to-Visible Upconversion Sensitized by Colloidal Nanocrystals. Nat. Photonics 2016, 10 (1), 31-34. https://doi.org/10.1038/nphoton.2015.226.

(17) VanOrman, Z. A.; Bieber, A. S.; Wieghold, S.; Nienhaus, L. Green-to-Blue Triplet Fusion Upconversion Sensitized by Anisotropic CdSe Nanoplatelets. Chem. Mater. 2020, 32 (11), 4734-4742. https://doi.org/10.1021/acs.chemmater.0c01354.

(18) Eaton, S. W.; Fu, A.; Wong, A. B.; Ning, C.-Z.; Yang, P. Semiconductor Nanowire Lasers. Nature Reviews Materials 2016, 1 (6), 1-11. https://doi.org/10.1038/natrevmats.2016.28.

(19) Quan, L. N.; Kang, J.; Ning, C.-Z.; Yang, P. Nanowires for Photonics. Chem. Rev. 2019, 119 (15), 9153-9169. https://doi.org/10.1021/acs.chemrev.9b00240.

(20) Tian, B.; Lieber, C. M. Nanowired Bioelectric Interfaces. Chem. Rev. 2019, 119 (15), 91369152. https://doi.org/10.1021/acs.chemrev.8b00795.

(21) Hochbaum, A. I.; Yang, P. Semiconductor Nanowires for Energy Conversion. Chem. Rev. 2010, 110 (1), 527-546. https://doi.org/10.1021/cr900075v.

(22) Zhang, S.-Y.; D. Regulacio, M.; Han, M.-Y. Self-Assembly of Colloidal One-Dimensional Nanocrystals. Chemical Society Reviews 2014, 43 (7), 2301-2323. https://doi.org/10.1039/C3CS60397K.

(23) Joarder, B.; Yanai, N.; Kimizuka, N. Solid-State Photon Upconversion Materials: Structural Integrity and Triplet-Singlet Dual Energy Migration. J. Phys. Chem. Lett. 2018, 9 (16), 4613-4624. https://doi.org/10.1021/acs.jpclett.8b02172.

(24) Lin, T.-A.; Perkinson, C. F.; Baldo, M. A. Strategies for High-Performance Solid-State Triplet-Triplet-Annihilation-Based Photon Upconversion. Advanced Materials 2020, 32 (26), 1908175. https://doi.org/10.1002/adma.201908175.

(25) Mase, K.; Okumura, K.; Yanai, N.; Kimizuka, N. Triplet Sensitization by Perovskite Nanocrystals for Photon Upconversion. Chem. Commun. 2017, 53 (59), 8261-8264. https://doi.org/10.1039/C7CC03087H.

(26) Huang, Z.; Li, X.; Yip, B. D.; Rubalcava, J. M.; Bardeen, C. J.; Tang, M. L. Nanocrystal Size and Quantum Yield in the Upconversion of Green to Violet Light with CdSe and Anthracene $\begin{array}{lllll}\text { Derivatives. } \quad \text { Chem. } & \text { Mater. 2015, } 27 \text { (21), }\end{array}$ https://doi.org/10.1021/acs.chemmater.5b03731.

(27) Gray, V.; Xia, P.; Huang, Z.; Moses, E.; Fast, A.; Fishman, D. A.; Vullev, V. I.; Abrahamsson, M.; Moth-Poulsen, K.; Tang, M. L. CdS/ZnS Core-Shell Nanocrystal 
Photosensitizers for Visible to UV Upconversion. Chem. Sci. 2017, 8 (8), 5488-5496. https://doi.org/10.1039/C7SC01610G.

(28) Okumura, K.; Yanai, N.; Kimizuka, N. Visible-to-UV Photon Upconversion Sensitized by Lead Halide Perovskite Nanocrystals. Chem. Lett. 2019. https://doi.org/10.1246/cl.190473.

(29) He, S.; Luo, X.; Liu, X.; Li, Y.; Wu, K. Visible-to-Ultraviolet Upconversion Efficiency above 10\% Sensitized by Quantum-Confined Perovskite Nanocrystals. J. Phys. Chem. Lett. 2019, 10 (17), 5036-5040. https://doi.org/10.1021/acs.jpclett.9b02106.

(30) Xia, P.; Raulerson, E. K.; Coleman, D.; Gerke, C. S.; Mangolini, L.; Tang, M. L.; Roberts, S. T. Achieving Spin-Triplet Exciton Transfer between Silicon and Molecular Acceptors for Photon Upconversion. Nature Chemistry 2020, 12 (2), 137-144. https://doi.org/10.1038/s41557-019-0385-8.

(31) Lai, R.; Wu, K. Red-to-Blue Photon Upconversion Based on a Triplet Energy Transfer Process Not Retarded but Enabled by Shell-Coated Quantum Dots. J. Chem. Phys. 2020, 153 (11), 114701. https://doi.org/10.1063/5.0023052.

(32) Mahboub, M.; Maghsoudiganjeh, H.; Pham, A. M.; Huang, Z.; Tang, M. L. Triplet Energy Transfer from $\mathrm{PbS}(\mathrm{Se})$ Nanocrystals to Rubrene: The Relationship between the Upconversion Quantum Yield and Size. Adv. Funct. Mater. 2016, 26 (33), 6091-6097. https://doi.org/10.1002/adfm.201505623.

(33) Luo, X.; Lai, R.; Li, Y.; Han, Y.; Liang, G.; Liu, X.; Ding, T.; Wang, J.; Wu, K. Triplet Energy Transfer from CsPbBr3 Nanocrystals Enabled by Quantum Confinement. J. Am. Chem. Soc. 2019, 141 (10), 4186-4190. https://doi.org/10.1021/jacs.8b13180.

(34) Peng, Z. A.; Peng, X. Formation of High-Quality CdTe, CdSe, and CdS Nanocrystals Using $\mathrm{CdO}$ as Precursor. J. Am. Chem. Soc. 2001, 123 (1), 183-184. https://doi.org/10.1021/ja003633m.

(35) He, S.; Lai, R.; Jiang, Q.; Han, Y.; Luo, X.; Tian, Y.; Liu, X.; Wu, K. Engineering Sensitized Photon Upconversion Efficiency via Nanocrystal Wavefunction and Molecular Geometry. Angewandte Chemie International Edition $n / a \quad$ (n/a). https://doi.org/10.1002/anie.202009066.

(36) Mahboub, M.; Huang, Z.; Tang, M. L. Efficient Infrared-to-Visible Upconversion with Subsolar Irradiance. Nano Lett. 2016, $16 \quad$ (11), 7169-7175. https://doi.org/10.1021/acs.nanolett.6b03503.

(37) Kagan, C. R.; Murray, C. B.; Nirmal, M.; Bawendi, M. G. Electronic Energy Transfer in CdSe Quantum Dot Solids. Phys. Rev. Lett. 1996, 76 (9), 1517-1520. https://doi.org/10.1103/PhysRevLett.76.1517.

(38) Chou, K. F.; Dennis, A. M. Förster Resonance Energy Transfer between Quantum Dot Donors and Quantum Dot Acceptors. Sensors 2015, 15 (6), 13288-13325. https://doi.org/10.3390/s150613288.

(39) Huang, Z.; Tang, M. L. Designing Transmitter Ligands That Mediate Energy Transfer between Semiconductor Nanocrystals and Molecules. J. Am. Chem. Soc. 2017, 139 (28), 9412-9418. https://doi.org/10.1021/jacs.6b08783.

(40) Dexter, D. L. A Theory of Sensitized Luminescence in Solids. J. Chem. Phys. 1953, 21 (5), 836-850. https://doi.org/10.1063/1.1699044.

(41) Zhou, Y.; Castellano, F. N.; Schmidt, T. W.; Hanson, K. On the Quantum Yield of Photon Upconversion via Triplet-Triplet Annihilation. ACS Energy Lett. 2020, 5 (7), 2322-2326. https://doi.org/10.1021/acsenergylett.0c01150. 
(42) Rigsby, E. M.; Miyashita, T.; Jaimes, P.; Fishman, D. A.; Tang, M. L. On the SizeDependence of CdSe Nanocrystals for Photon Upconversion with Anthracene. J. Chem. Phys. 2020, 153 (11), 114702. https://doi.org/10.1063/5.0017585.

(43) Kriegel, I.; Scotognella, F.; Soavi, G.; Brescia, R.; Rodríguez-Fernández, J.; Feldmann, J.; Lanzani, G.; Tassone, F. Delayed Electron Relaxation in CdTe Nanorods Studied by Spectral Analysis of the Ultrafast Transient Absorption. Chemical Physics 2016, 471, 3945. https://doi.org/10.1016/j.chemphys.2015.08.002.

(44) Klimov, V. I. Optical Nonlinearities and Ultrafast Carrier Dynamics in Semiconductor Nanocrystals. J. Phys. Chem. B 2000, $104 \quad$ (26), 6112-6123. https://doi.org/10.1021/jp9944132.

(45) Mongin, C.; Garakyaraghi, S.; Razgoniaeva, N.; Zamkov, M.; Castellano, F. N. Direct Observation of Triplet Energy Transfer from Semiconductor Nanocrystals. Science 2016, 351 (6271), 369-372. https://doi.org/10.1126/science.aad6378.

(46) He, S.; Lai, R.; Jiang, Q.; Han, Y.; Luo, X.; Tian, Y.; Liu, X.; Wu, K. Engineering Sensitized Photon Upconversion Efficiency via Nanocrystal Wavefunction and Molecular Geometry. Angewandte Chemie International Edition 2020, 59 (40), 17726-17731. https://doi.org/10.1002/anie.202009066.

(47) Monguzzi, A.; Mezyk, J.; Scotognella, F.; Tubino, R.; Meinardi, F. Upconversion-Induced Fluorescence in Multicomponent Systems: Steady-State Excitation Power Threshold. Phys. Rev. B 2008, 78 (19), 195112. https://doi.org/10.1103/PhysRevB.78.195112.

(48) De Roo, J.; Huang, Z.; Schuster, N. J.; Hamachi, L. S.; Congreve, D. N.; Xu, Z.; Xia, P.; Fishman, D. A.; Lian, T.; Owen, J. S.; Tang, M. L. Anthracene Diphosphate Ligands for CdSe Quantum Dots; Molecular Design for Efficient Upconversion. Chem. Mater. 2020, 32 (4), 1461-1466. https://doi.org/10.1021/acs.chemmater.9b04294.

(49) Huang, Z.; Simpson, D. E.; Mahboub, M.; Li, X.; Tang, M. L. Ligand Enhanced Upconversion of Near-Infrared Photons with Nanocrystal Light Absorbers. Chem. Sci. 2016, 7 (7), 4101-4104. https://doi.org/10.1039/C6SC00257A.

(50) Wieghold, S.; Bieber, A. S.; VanOrman, Z. A.; Nienhaus, L. Influence of Triplet Diffusion on Lead Halide Perovskite-Sensitized Solid-State Upconversion. J. Phys. Chem. Lett. 2019, 10 (13), 3806-3811. https://doi.org/10.1021/acs.jpclett.9b01526. 
TOC Graphic

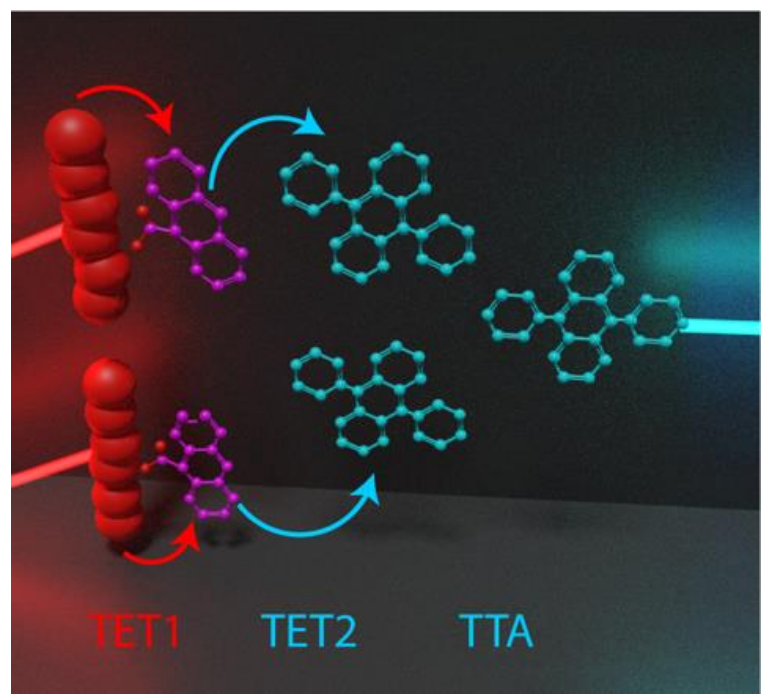

